\title{
Least Concave Ordinal Utility Function and the Marshalian Cardinal Utility
}

\author{
TARADAS BANDYOPADHYAY \\ University of California, Riverside
}

TAPAN BISWAS

University of Hull

\begin{abstract}
This paper examines the conditions under which the Marshallian type of cardinal utility function can be derived from a class of ordinal utility functions.

Keywords:

JEL Classifications:
\end{abstract}

\section{Introduction}

Although Alfred Marshall (1990) developed his theory of demand based on maximizing a cardinal utility function, in to-day's text books a Marshallian demand function is derived from the exercise of maximizing an ordinal utility function. The purpose of this short paper is to present the condition under which the Marshallian type of cardinal utility function can be derived from a class of ordinal utility functions. ${ }^{1}$ In contrast to a Paretian consumer who begins the day with a predetermined income or an amount of money to spend, a Marshallian consumer starts shopping for the day with a predetermined rate of exchange between money and utility. ${ }^{2}$ Marshall writes: "... we assume that the marginal utility of money to the

${ }^{1}$ The relationship between an ordinal utility function and the Von Neumann-Morgenstern's cardinal utility function was investigated by Baumol (1958).

${ }^{2}$ A few years after the publication of Principles, Sanger (1895) writes in a survey article: "Professor Marshall assumes that $\mathrm{m}$ [the marginal utility of money] remains constant. .According to Professor Walras, the individual spends an amount of money $\mathrm{M}$, while Professor Marshall makes him spend $\mathrm{M}+\delta \mathrm{M}$ where $\delta \mathrm{M}$ is evidently given by $\delta \mathrm{M}=\mathrm{r}_{\mathrm{a}} \Delta \mathrm{p}_{\mathrm{a}}+\mathrm{p}_{\mathrm{a}} \delta \mathrm{r}_{\mathrm{a}}+\mathrm{r}_{\mathrm{b}} \Delta \mathrm{p}_{\mathrm{b}}+\mathrm{p}_{\mathrm{b}} \delta \mathrm{r}_{\mathrm{b}}+\mathrm{r}_{\mathrm{c}} \Delta \mathrm{p}_{\mathrm{c}}+\mathrm{p}_{\mathrm{c}} \delta \mathrm{r}_{\mathrm{c}}{ }^{\prime \prime}(\mathrm{pp}$ 125).

Here $\delta \mathrm{M}$ is the change in money expenditure when the prices change, but to the consumer the marginal utility of money remains the same. Marshall referred to this section of Sanger's article in the 8th edition of his "Principles" (pp 109). It seems that Marshall agreed to this interpretation.

(C) 2011 Taradas Bandyopadhyay and Tapan Biswas. Licensed under the Creative Commons Attribution - Noncommercial 3.0 Licence (http: / / creativecommons . org/licenses/bync/3.0/. Available at http://rofea.org. 
individual purchaser is the same throughout" (p. 842). ${ }^{3}$ Hick's (1946) interpretation of the constancy of marginal utility of money is that the rate of substitution between commodities and money is independent of the amount of money at the disposal of a consumer. In his framework, the constancy of marginal utility of money implies that an ordinal utility function is increasing in money. Assuming that the utility function is not defined over the commodity bundles that are smaller than the bundles of goods and services that are minimally necessary for bare survival, the constancy of marginal utility of money would imply that a consumer can attain any given level of utility with a subsistence bundle and an appropriate amount of money. With these in mind we have established the relationship between a utility function that preserves the convex preordering on the commodity space and a Marshallian cardinal utility function ${ }^{4}$

\section{Preliminaries}

Let a commodity space $Y$ be a nonempty convex set in a real space $\Re^{n}$ and a preference relation $R$ be a complete preorder, i.e. a complete, reflexive and transitive preference relation on $Y$. We assume that a strictly quasi-concave representation of the preorder is known to exist. We say that a real valued function $u$ on $Y$ represents $R$ if $\left[y_{1} R y_{2}\right]$ is equivalent to $\left[u\left(y_{1}\right) \geq\right.$ $u\left(y_{2}\right)$ ]. We assume that $u(y)$ is strictly quasi-concave, twice differentiable real valued function on $Y$ representing $R$. We also assume that $Y$ has a lower bound with $y_{n} \geq 0$ where, $y_{n}$ is the $n^{\text {th }}$ component of the vector $y \in Y$. We will interpret $y_{n}$ as money.

Let $\left(\tilde{y}, y_{n}\right) \in Y$ where $\tilde{y} \in \tilde{Y} \subseteq \mathfrak{R}^{\mathrm{n}-1}$. Clearly, $\tilde{Y}$ is the projection of $Y$ onto the space of n-1 commodities. We assume that, if $\left(\tilde{y}, y_{n}\right) \in Y$, then $(\tilde{y}, 0) \in Y$ and $\tilde{Y}=(\tilde{y} \mid(\tilde{y}, 0) \in Y)$ has a lower bound. Very small quantities of all n-1 commodities may be inadequate to permit the consumer to survive. We assume that there exists a set $S \subseteq \tilde{Y}$, the bare survival set, such that $s$ $\in S$ implies $u(s, 0)=\min u\left((\tilde{y}, 0), \tilde{y} \in \tilde{Y}\right.$, i.e., for $\tilde{y}^{\prime}<\mathrm{s}, u\left(\tilde{y}^{\prime}, 0\right)$ is not defined.

Let $U$ be the set of continuous, concave, real-valued functions on $Y$ representing $R$. If there exists a $u^{*} \in U$ such that $u^{*}=f(u), u \in U$, and $f$ is a strictly concave function, then $u^{*}$ is said to be more concave than $u$. In that case, the set $U$ is preordered by the relation $u^{*}$ is more

\footnotetext{
${ }^{3}$ This is justified "on the assumption, which underlies our whole reasoning, that his expenditure on any one thing, as, for instance, tea, is only a small part of his whole expenditure" (ibid). Interested readers are also referred to pages 132-135 of Marshall's Principles.

${ }^{4}$ Hicks (1946, pp 26) wrote: "What is meant by the marginal utility of money being constant? Making our translation, it would appear to mean that changes in the consumer's supply of money (that is, with respect to the problem in hand, his income) will not affect the marginal rate of substitution between money and any particular commodity $\mathrm{x}$. The demand for $\mathrm{x}$ is therefore independent of income. His demand for any commodity is independent of income. It will appear in what follows that this is actually what the constancy of the marginal utility of money did mean for Marshall; not that he really supposed that peoples demand for commodities do not depend upon their incomes, but that in his theory of demand and price he generally neglected the income side."
} 


\section{BANDYOPADHYAY, BISWAS Utility Functions}

concave than $u$ defined by a real-valued strictly concave function $f$ on $u(Y)$. The function $f$ is increasing and continuous since it maps the interval $u(Y)$ onto the interval $u^{*}(Y)$. If it is not possible for $u^{*} \in U$ to be a strictly concave transformation of any $u \in U$, then $\mathrm{u}^{*}$ is said to be a least concave function representing $R$ on $Y$. The linear transformation of $u^{*}$ also belongs to the class of least concave functions representing $R$ on $Y$.

\section{Ordinal Utility Functions and a Marshallian Consumer}

We now present three conditions that would establish the relation between an ordinal utility function and a Marshallian cardinal utility function. Every one of these conditions follows from a Marshallian view of a consumer who begins the day with a predetermined rate of exchange between money and utility.

Axiom 1. $u\left(\tilde{y}, y_{n}\right)$ is a strictly increasing function in $y_{n}$.

Axiom 2. For any $y \in Y$, there exists a real number $\mu$ such that $u(y)=u(s, \mu)$ for every $s \in S$ $\subseteq \tilde{Y}$.

Given axiom $1, \mu$ is clearly unique for any $y$. Axiom 2 says that a consumer can attain any given level of utility with a subsistence consumption bundle and an appropriate amount of $y_{n}$. Given the assumption, if $\left(\tilde{y}, y_{n}\right) \in Y$, then $(\tilde{y}, 0) \in Y$ and $\tilde{Y}=(\tilde{y} \mid(\tilde{y}, 0) \in Y)$ has a lower bound. Then, axioms 1 and 2 imply that the strictly quasi-concave, twice differentiable real valued function $u$ attains a minimum on $Y$.

Now we introduce a money metric function. By axiom 1, there exists a real valued function $\psi$ which maps the elements of $Y$ to a real line such that for all $y \in Y, \mu=\psi(y)$, where $u(y)=u(s, \mu)$. Thus, for any given subsistence bundle of $n$-1 commodities, $s$, we can define a function $\psi(y), y \in Y$. In other words, by axiom 2, for any subsistence bundle $s \in S$, the real number $\mu$ is defined as the money equivalent of $y$, and $\psi(y)$ is called the money metric function. It means that a consumer is indifferent between a bundle $\left(\tilde{y}, y_{n}\right)$ and a bundle $(s, \mu)$.

Axiom 3. For $y, y^{\prime} \in Y$, where $y_{n} \geq y_{n}{ }^{\prime}$ and $y_{i}=y_{i}{ }^{\prime}$ for all $i \neq n, \psi(y)-\psi\left(y^{\prime}\right)=y_{n}-y_{n}{ }^{\prime}$.

The axiom 3 implies that the rate of substitution between $\tilde{y}$ (where $\tilde{y} \in \tilde{Y} \subseteq \Re^{\mathrm{n}-1}$ ) and $y_{n}$ is independent of the magnitude of $y_{n}{ }^{5}$ Since $u\left(\tilde{y}, y_{n}\right)$ is increasing in $y_{n}$, once again by axiom $3, u(s, \mu)$ is increasing in $\mu$.

${ }^{5}$ Utility functions, linear in money, have been discussed by many authors, e.g. Shapley and Shubik (1966). 
Review of Economic Analysis 4 (2012) 9-16

THEOREM 1. (1.1) $\psi\left(\tilde{y}, y_{n}\right)$ is quasi-linear in $y_{n}$;

(1.2) $\psi(\tilde{y}, 0)$ is a single-valued function; and

(1.3) $\psi(\tilde{y}, 0)$ preserves the preference ordering on $\tilde{Y}$ generated by $u$ on $Y$.

\section{PROOF.}

(1.1) Given axiom $3, \psi\left(\tilde{y}, y_{n}\right)-\psi(\tilde{y}, 0)=y_{n}$. Hence, $\psi\left(\tilde{y}, y_{n}\right)=\psi(\tilde{y}, 0)+y_{n}$.

(1.2) To the contrary, suppose $\psi(\tilde{y}, 0)$ assumes two different values $\mu_{1}$ and $\mu_{2}$, such that $\mu_{1}>$ $\mu_{2}$. Then, by axiom $2, u(\tilde{y}, 0)=u\left(s, \mu_{1}\right)$, and $u(\tilde{y}, 0)=u\left(s, \mu_{2}\right)$. Clearly, $u\left(s, \mu_{1}\right)=u\left(s, \mu_{2}\right)$ and $\mu_{1}>\mu_{2}$ together contradicts the fact that $u(s, \mu)$ is an increasing function in $\mu$ which follows from axioms 1 and 3.

(1.3) We have to establish that the preference ordering of vectors in $\tilde{Y}$ is independent of the level of $y_{n}$. It is left to the reader to check that for $\tilde{y}, \tilde{y}^{*} \in \tilde{Y}$, if $\psi(\tilde{y}, 0) \geq \psi\left(\tilde{y}^{*}, 0\right)$ then $u(\tilde{y}, 0) \geq$ $u\left(\tilde{y}^{*}, 0\right)$. What remains is to show that if $u(\tilde{y}, 0) \geq u\left(\tilde{y}^{*}, 0\right)$ then $\psi(\tilde{y}, 0) \geq \psi\left(\tilde{y}^{*}, 0\right)$. Suppose $u(\tilde{y}, 0) \geq u\left(\tilde{y}^{*}, 0\right)$. By axiom 2, there exists a survival bundle $s \in S$ and $\mu_{1}, \mu_{2}$ such that $u(\tilde{y}, 0)=$ $u\left(s, \mu_{1}\right)$, and $u\left(\tilde{y}^{*}, 0\right)=u\left(s, \mu_{2}\right)$. Since $u(s, \mu)$ is an increasing function in $\mu$, therefore $\mu_{1} \geq \mu_{2}$. By definition, $\mu_{1}=\psi(\tilde{y}, 0)$ and $\mu_{2}=\psi\left(\tilde{y}^{*}, 0\right)$. Hence, $\psi(\tilde{y}, 0) \geq \psi\left(\tilde{y}^{*}, 0\right)$. This completes the proof.

Next utilizing the money metric function, $\psi(y)$, we construct the Marshallian utility function. It is clear from theorem 1 , that $\psi(\tilde{y}, 0)$ is consistent with $u(y)$. Since $u($.$) is a utility function$ defined on $Y, \psi(\tilde{y}, 0)$ may be treated as a utility function defined on $\tilde{Y}$. Now by (1.1) of theorem 1 , one unit of utility is equivalent to one unit of $y_{n}$, i.e., the exchange between $y_{n}$ and utility is fixed. Thus one may consider any linear transformation of $\psi(\tilde{y}, 0)$ as the utility function, i.e.,

$$
V=a+\lambda \psi(\tilde{y}, 0), \text { with } \lambda>0 .
$$

For $\left(\tilde{y}, y_{n}\right) \in Y$, this relation can be written as

$$
V=a+\lambda \psi\left(\tilde{y}, y_{n}\right) .
$$

Since $\mu\left(\tilde{y}, y_{n}\right)$ is quasi-linear in $y_{n}$, and also since the exchange between $y_{n}$ and utility is fixed,

$$
V=a+v(\tilde{y})+\lambda y_{n},
$$

where $v(\tilde{y})=\lambda \psi(\tilde{y}, 0) . V$ is a linear transformation of the money metric function and will be called the Marshallian utility function where the marginal utility of money is $\lambda$. Clearly, the constancy of marginal utility of money is crucial in the construction of a Marshallian utility 


\section{BANDYOPADHYAY, BISWAS Utility Functions}

function such as $V$. The result below now shows that any linear transformation of $\psi(\tilde{y}, 0)$ is strictly concave if and only if $u(y)$ is strictly quasi-concave.

THEOREM 2. The function $v(\tilde{y})$ is strictly concave on $\tilde{Y}$ if and only if $u\left(\tilde{y}, y_{n}\right)$ is strictly quasiconcave on $Y^{6}$

PROOF. We first establish the sufficiency part. Since by definition, $v(\tilde{y})=\lambda \psi(\tilde{y}, 0)$, it will suffice to show that $\mu(\tilde{y})$ is strictly concave. Suppose $u\left(\tilde{y}, y_{n}\right)$ is strictly quasi-concave. For distinct $\tilde{y}^{1}, \tilde{y}^{2} \in \tilde{Y}$ suppose $\psi\left(\tilde{y}^{1}, 0\right) \leq \psi\left(\tilde{y}^{2}, 0\right)$. We have to show that for $\theta \in(0,1)$,

$$
\theta \psi\left(\tilde{y}^{1}, 0\right)+(1-\theta) \psi\left(\tilde{y}^{2}, 0\right)<\psi\left(\tilde{y}^{*}, 0\right),
$$

where $\tilde{y}^{*}=\theta \tilde{y}^{1}+(1-\theta) \tilde{y}^{2}$. Consider $y_{n}$ such that $\psi\left(\tilde{y}^{2}, 0\right)=\psi\left(\tilde{y}^{1}, 0\right)+y_{n}$. By construction, $u\left(\tilde{y}^{1}, y_{n}\right)=u\left(\tilde{y}^{2}, 0\right)$. Since $u\left(\tilde{y}^{1}, y_{n}\right)$ is strictly quasi-concave, $u\left(\tilde{y}^{1}, y_{n}\right)=u\left(\tilde{y}^{2}, 0\right)<u\left(\tilde{y}^{*}, \theta y_{n}\right)$. Thus,

$$
\psi\left(\tilde{y}^{1}, 0\right)+y_{n}=\psi\left(\tilde{y}^{2}, 0\right)<\psi\left(\tilde{y}^{*}, \theta y_{n}\right) .
$$

Now taking the convex combinations, we obtain,

$$
\theta\left(\psi\left(\tilde{y}^{1}, 0\right)+y_{n}\right)+(1-\theta) \psi\left(\tilde{y}^{2}, 0\right)<\psi\left(\tilde{y}^{*}, 0\right)+\theta y_{n},
$$

which gives us,

$$
\theta \psi\left(\tilde{y}^{1}, 0\right)+(1-\theta) \psi\left(\tilde{y}^{2}, 0\right)<\psi\left(\tilde{y}^{*}, 0\right)
$$

Next we consider the necessity part. For two distinct bundles, $\left(\tilde{y}^{1}, y_{n}{ }^{1}\right),\left(\tilde{y}^{2}, y_{n}{ }^{2}\right) \in Y$, suppose $u\left(\tilde{y}^{1}, y_{n}{ }^{1}\right)=u\left(\tilde{y}^{2}, y_{n}{ }^{2}\right)$. We have to show that for $\theta \in(0,1)$

$$
u\left(\tilde{y}^{1}, y_{n}^{1}\right)=u\left(\tilde{y}^{2}, y_{n}^{2}\right)<u\left(\tilde{y}^{*}, \theta y_{n}{ }^{*}\right)
$$

where

$$
\tilde{y}^{*}=\theta \tilde{y}^{1}+(1-\theta) \tilde{y}^{2} \text { and } y_{n}{ }^{*}=\theta y_{n}{ }^{1}+(1-\theta) y_{n}{ }^{2}
$$

By construction,

$$
\psi\left(\tilde{y}^{1}, 0\right)+y_{n}{ }^{1}=\psi\left(\tilde{y}^{2}, 0\right)+y_{n}{ }^{2} .
$$

Without loss of generality, suppose $y_{n}{ }^{1} \geq y_{n}{ }^{2}$. This implies that $\psi\left(\tilde{y}^{1}, 0\right) \leq \psi\left(\tilde{y}^{2}, 0\right)$. Since $\psi(\tilde{y}$, $0)$ is strictly concave,

${ }^{6}$ For the sake of simplicity of the proof, we have assumed strict concavity and strict convexity. To see the connection of our result with the literature on convex analysis recall a result of Debreu and Koopmans (1982) which shows that if a real valued function $f$ (defined on the product of $\mathrm{n}$ finitedimensional open convex sets $\left.Y_{i}, i=1,2, \ldots, n\right)$ is quasi-convex such that $f=\sum f_{i}$, where $f_{i}$ is defined on the factor set $Y_{i}\left(i=1,2, \ldots, n\right.$,), then every $f_{i}$ is continuous and with at most one exception every $f_{i}$ is convex. Note that given the assumptions 1,2 and 3, the exception would not arise in our case. 
Review of Economic Analysis 4 (2012) 9-16

$$
\theta \psi\left(\tilde{y}^{1}, 0\right)+(1-\theta) \psi\left(\tilde{y}^{2}, 0\right)<\psi\left(\tilde{y}^{*}, 0\right) .
$$

Clearly,

$$
\theta\left(\psi\left(\tilde{y}^{1}, 0\right)+y_{n}^{1}\right)+(1-\theta)\left(\psi\left(\tilde{y}^{2}, 0\right)+y_{n}^{2}\right)<\psi\left(\tilde{y}^{*}, 0\right)+y_{n}^{*} .
$$

Since,

$$
\psi\left(\tilde{y}^{1}, 0\right)+y_{n}{ }^{1}=\psi\left(\tilde{y}^{2}, 0\right)+y_{n}{ }^{2}
$$

therefore,

$$
\begin{aligned}
& \psi\left(\tilde{y}^{1}, 0\right)+y_{n}{ }^{1}<\psi\left(\tilde{y}^{*}, 0\right)+y_{n}{ }^{*} \text { and } \\
& \psi\left(\tilde{y}^{2}, 0\right)+y_{n}{ }^{2}<\psi\left(\tilde{y}^{*}, 0\right)+y_{n}{ }^{*} .
\end{aligned}
$$

By theorem 1,

$$
u\left(\tilde{y}^{1}, y_{n}{ }^{1}\right)=u\left(\tilde{y}^{2}, y_{n}^{2}\right)<u\left(\tilde{y}^{*}, y_{n}^{*}\right) .
$$

This completes the proof of the theorem.

Now we show that if a preference preorder is represented by a continuous, strictly quasiconcave, real valued utility function, then a least concave utility function representing the preorder is indeed a Marshallian cardinal utility function. First we establish that $V$ is a least concave utility function.

THEOREM 3. A Marshallian utility function such as $V$ is a least concave utility function that preserves the convex preordering $R$ on $Y$ represented by $u\left(\tilde{y}, y_{n}\right)$.

PROOF. For the sake of simplicity, let $a=0$ and $\lambda=1$. We shall prove that the function

$$
V=\psi(\tilde{y}, 0)+y_{n}
$$

is a least concave utility function. Let $V^{*}\left(\tilde{y}, y_{n}\right)$ be a concave function and $f$ be a strictly concave function such that $V=f\left(V^{*}\right)$. Clearly. $f^{\prime}>0$. Since $V^{*}\left(\tilde{y}, y_{n}\right)$ is concave, the second order partial differentiation of $V^{*}$ with respect to $y_{n}, V^{*}{ }_{n n}$ exits almost everywhere and must be non-positive. By concavity, $f$ " also exists almost everywhere. Now given

$$
\psi(\tilde{y}, 0)+y_{n}=f\left(V^{*}\left(\tilde{y}, y_{n}\right)\right)
$$

we obtain

$$
0=f^{\prime \prime} V_{\mathrm{n}}^{*}+f^{\prime} \mathrm{V}_{\mathrm{nn}}
$$




\section{BANDYOPADHYAY, BISWAS Utility Functions}

by taking second order partial differentiation with respect to $y_{n}$, wherever possible. Given $f^{\prime}>$ 0 and $V^{*}{ }_{n n} \leq 0, f^{\prime \prime} \geq 0$. By theorem $1, V^{*}{ }_{n}>0$. This is a contradiction with the assumption that $f$ is a strictly concave function. This completes the proof of our theorem.

Finally, recall the results of Debreu (1977), Kannai $(1974,1980)$ and Debreu-Koopmans (1982) that if a preference preorder is represented by a continuous, concave, real valued function, then a least concave function representing the preorder is a cardinal utility. Utilizing this result it is immediate that the utility function such as $V$ is indeed a cardinal utility function. ${ }^{7}$

The utility function such as $V$ is an outcome of a Marshallian view of a consumer. Although a Marshallian consumer starts shopping for the day with a predetermined rate of exchange between money and utility in contrast to a Paretian consumer who begins with a predetermined level of expenditure, in equilibrium, the marginal utility of money must be equal to the marginal utility of expenditure. ${ }^{8}$ Following Hicks (1956, pp 12-15), utilizing the function $V(y)$, Biswas (1977) factorized the price effect into direct and indirect effects, which is conceptually related to the substitution and income effects of a Paretian consumer who starts shopping with a predetermined level of expenditure. The direct effect tells us the effect on the consumption of a commodity due to change in price, keeping the marginal utility of money constant. In comparison, the substitution effect shows the effect on the consumption of a commodity due to change in price, keeping the real income or expenditure constant. The indirect effect is the product of the effect on the consumption due to change in expenditure keeping prices constant and the change in expenditure due to change in price for a given value of the marginal utility of money. ${ }^{9}$ In contrast, the income effect of a Paretian consumer is the

7 The reader should note that there is a variation in the definition of least concavity, however, it does not affect our remark.

8 Note that throughout the "Principles" (including the Mathematical Appendix), Marshall never mentioned any income constraint. He wrote: "A prudent person will endeavor to distribute his means between all their several uses, present and future, in such a way that they will have in each the same marginal utility" (pp 100). This means that the utility gained from spending one unit of money for any commodity in any period, present or future should be the same (which is equal to the marginal or the shadow utility of monetary assets). This is obviously an optimality condition for a simple inter-temporal utility maximization problem. In a dynamic utility maximization model, the marginal or shadow utility of money depends on the stream of current and expected future incomes and on the time profile of current and expected prices. If the planning horizon lies very far ahead, from a well known result in dynamic optimization theory it follows that a small (may be finite in continuous time analysis) perturbation in income or prices, which is thought to be temporary in character, will have a negligible impact on the shadow price or marginal utility of monetary wealth. In other words, any small variation in income, which is supposed to last only for a short period, will have virtually no repercussion on the marginal utility of money and hence on the amount of commodities purchased.

9 Given the decision problem of a Marshallian consumer: maximize $L=V(y)-\lambda p y$, where $y$ and $p$ are the consumption and the price vectors respectively, and $\lambda$ is the marginal utility of money. The first order condition for a maximum is $V_{i}=\lambda p_{i}$ which, for a given $p$, gives $\mathrm{H} \delta y=\delta \lambda$, where $\mathrm{H}$ is the Hessian of the utility function $V(y)$. It is immediate that one can obtain the comparative static result of a price 
Review of Economic Analysis 4 (2012) 9-16

product of the effect on the consumption of a commodity due to change in expenditure keeping the prices constant and the change in expenditure due to change in price of a commodity for a given level of income in terms of utility. In fact, the constancy of the marginal utility of money as opposed to the constancy of a predetermined level of income in terms of-utility explains the Giffen good phenomena of a Marshallian consumer. It is obvious that if $V(y)$ is negative definite everywhere, any Paretian equilibrium is also an equilibrium of a Marshallian consumer.

\section{References}

Baumol, W. J. (1958), The Cardinal Utility Which Is Ordinal, Economic Journal 68, 665-672.

Biswas, T. (1977), The Marshallian Consumer, Economica 87, 47-56.

Debreu, G. (1976), Least Concave Utility Functions, JournaI of Mathematical Economics 3, 121-129.

Debreu, G. and Koopmans, T. C. (1982), Additively Decomposed Quasiconvex Functions, Mathematical Programming 24, 1-38.

Friedman, M. (1949), The Marshallian Consumer, Journal of Political Economy 57, 463-495.

Hicks, J. R. (1946), Value and Capital, 2nd edition, London: Oxford University Press.

Hicks, J. R. (1956), Revision of Demand Theory, London: Oxford University Press.

Kannai, Y. (1974), Approximation of Convex Preferences, Journal of Mathematical Economics 1, 101-106.

Kannai, Y. (1980), The ALEP Definition of Complementarity and Least Concave Utility Functions, Journal of Economic Theory 22, 115-1 17.

Marshall, A. (1890), Principles of Economics (8th ed., 1920), London: Macmillan.

Samuelson, P. A. (1942), Constancy of the Marginal Utility of Income, in 0. Lange et a1

(edited), Studies in Mathematical Economics and Econometrics, Chicago: Chicago University Press.

Sanger, C. P. (1895), Recent Contribution in Mathematical Economics, Economic Journal 5, 113-128.

Shapley, L. S. and Shubik, M. (1966), Quasicore in a Monetary Economy with Nonconvex Preference, Econometrica 34, 805-827.

change of commodity $i$ as: $\left(\delta y_{i} / \delta p_{i}\right)=\left(\lambda\left|\mathrm{H}_{i i}\right| /|\mathrm{H}|\right)-\left(\delta \mathrm{E} / \delta p_{i}\right)_{\lambda=\lambda^{0}}\left(\delta y_{i} / \delta \mathrm{E}^{*}\right)_{p=p}{ }^{0}$, where $\mathrm{E}$ is the actual expenditure and $\mathrm{E}^{*}$ is the predetermined expenditure level. The first term of the right hand side is the direct effect of a price change and the second term is the indirect effect. 